\title{
La Importancia del Dominio del Idioma Ingles con Propositos Especificos en la Formacion Odontologica
}

\author{
Andrea Stephanie Bayas Bayas, $O D$ \\ Dennys Vladimir Tenelanda López, MA \\ Edison Fernando Bonifaz Aranda, MA \\ Natalia Gavilanes Bayas, OD \\ Universidad Nacional de Chimborazo, Ecuador
}

Doi:10.19044/esj.2019.v15n25p348 URL:http://dx.doi.org/10.19044/esj.2019.v15n25p348

\section{Resumen}

El presente estudio tuvo como objetivo determinar las necesidades, falencias y fortalezas de los profesionales en el campo de la odontología en relación al manejo de las cuatro destrezas del idioma inglés con propósitos específicos. Esta investigación fue de tipo descriptivo, de campo y de enfoque mixto, en el que se aplicó como técnica la encuesta y como instrumento un cuestionario dirigido a 26 docentes especialistas de la Carrera de Odontología de la Universidad Nacional de Chimborazo, Ecuador. Se evidenció que los especialistas poseían falencias sobre las cuatro destrezas en el idioma inglés, pues solamente el $7,7 \%$ manifestó tener un excelente nivel al escuchar y hablar, mientras que el 15, 4\% sobre el escribir y leer. Se probó la necesidad del diseño e implementación de un texto de trabajo para mejorar el dominio de destrezas y actualización de conocimientos del idioma inglés con propósitos odontológicos mediante un análisis de significancia unilateral hacia la derecha (prueba t de una muestra) en la que se comparó si los dominios de los docentes superaron el 0,70 ( $\mathrm{p}=0,00 ; 1-\mathrm{p} / 2=1 ; \mathrm{t}=4,34(25))$ en cuyo caso se mostró con un manejo de dominio menor al $70 \%$, la importancia de este aporte se mostró aceptable por parte de los docentes.

Palabras clave: Odontología, Inglés con propósitos específicos, Docentes de odontología, Destrezas 


\title{
Importance of English for Specific Purposes (ESP) in Dental Education
}

\author{
Andrea Stephanie Bayas Bayas, OD \\ Dennys Vladimir Tenelanda López, MA \\ Edison Fernando Bonifaz Aranda, MA \\ Natalia Gavilanes Bayas, OD \\ Universidad Nacional de Chimborazo, Ecuador
}

\begin{abstract}
This study aimed to determine the needs, shortcomings and strengths of professionals in the field of dentistry in relation to the management of the four English language skills for specific purposes. This research was of a descriptive, field and mixed approach, in which the survey was applied as a technique and as a tool a questionnaire addressed to 26 specialist professors of the Dentistry School of Universidad Nacional de Chimborazo. It was evidenced that the specialists have shortcomings about the four skills in the English language, because only $7.7 \%$ said they had an excellent level of listening and speaking, while $15.4 \%$ said they had an excellent level of listening and speaking. The need for the design and implementation of a working text to improve mastery of skills and updating of English language knowledge for dental purposes was proved through a one-way analysis to the right (sample $t$ test) in which it was compared if the domains of the professros exceeded $0.70(\mathrm{p}=0.00 ; 1-\mathrm{p} / 2=1 ; \mathrm{t}=4.34(25))$ in which case it was shown with a domain management of less than $70 \%$, the importance of this contribution was acceptable by the professors.
\end{abstract}

Keywords: Dentistry, English for Specific Purposes, Teachers, Skills

\section{Introducción}

El inglés con propósitos específicos se ha implementado en varias instituciones de educación superior enfocados a la formación de médicos (Sequeira, 2016), siendo una necesidad para la instrucción académica de futuros profesionales, la enseñanza del inglés es muy importante en el campo odontológico. Un profesional debe manejar las cuatro competencias básicas del idioma, como son: leer, hablar, escribir, escuchar, además de actualizar sus conocimientos por medio de la lectura de libros, artículos, revistas científicas y asistiendo o dando conferencias, dichas competencias distan mucho de la 
realidad de los profesionales encontrando que la mayoría presentan dificultades en el dominio de esta lengua extranjera.(Javid, 2011)

Según el ranking mundial más grande del mundo EF English Proficiency Index (EPI), Ecuador ocupa el puesto número 65 de 88 países o regiones, y el número 13 de 17 países en Latinoamérica, con un nivel de dominio bajo, además indica que América latina es la única región en el mundo que ha demostrado que el dominio del idioma inglés se ha reducido en el año 2018 , esto debido a la oferta de los sistemas educativos y su economía deficiente.(EF Education First, 2018)

En México, dentro de la Universidad Veracruzana, se realizó un estudio en 75 estudiantes para analizar su situación socioeducativa, en donde se encontró que el $52.30 \%$ de los estudiantes se considera poco hábil para leer en inglés textos académicos e información en internet, el $57.6 \%$ consideró tener el $25 \%$ o menos de dominio en la habilidad de lectura en inglés, al rendir un examen de ubicación al $77 \%$ de los estudiantes se los ubicó en el nivel 1 de 5 , en el examen el $80 \%$ de los estudiantes reprobó en la habilidad para la lectura, al analizar el número de docentes que solicitan a los estudiantes realizar lecturas en inglés, el 95\% de los estudiantes afirmó que los docentes no lo hacen.(Herrera \& Vallejo, 2013)

En Ecuador se realizó un estudio sobre 100 estudiantes de medicina de la Universidad Regional Autónoma de los Andes para conocer el dominio sobre las cuatro destrezas para el aprendizaje del idioma inglés, mediante un análisis cuantitativo se encontró que la destreza con mayor dominio es el Speaking con un valor máximo de 25,00 puntos, seguido por el Writing con 22,00 puntos, mientras que las destrezas en Reading y Listening indican un dominio muy deficiente con 16, 34 y 4,65 puntos respectivamente, además se evaluó el nivel de dominio del inglés con propósitos específicos y se encontró que el $49 \%$ obtuvo de entre 0 a 20 puntos, el $21 \%$ entre 21 a 40 puntos, demostrando que la mayoría carece de dominio o presenta un dominio escaso sobre este idioma. (Montes De Oca \& Morete, 2017)

En Europa, en la Universidad de Vilnia en Lituania, se realizó un estudio de alto impacto sobre 276 estudiantes, con la finalidad de conocer sus percepciones sobre la necesidad de un curso de inglés al final de sus estudios en el campo de las ciencias biomédicas, el $42 \%$ afirmó la importancia de estos cursos, el $37 \%$ tuvo dudas y el 26\% respondió negativamente, el $68 \%$ afirmó que el tipo de curso que necesitan es sobre inglés aplicado a su profesión, al averiguar si leen literatura en inglés, se evidenció que el 46\% lee libros de texto mientras que el 55\% lee literatura adicional, ya que la mayoría señaló al inglés como su primera lengua extranjera pero se halló que el $68 \%$ carece de habilidad lectora.(Rozgienè \& Trečiokaitè, 2019)

En Japón un estudio afirma que las clases de medicina y odontología son dictadas únicamente en japonés, pero gracias a la globalización de las 
profesiones, el Ministerio de Educación, Cultura, Deportes, Ciencia y Tecnología presentó la normativa de reforma del Sistema de Educación Superior con la introducción del inglés en la malla curricular de las profesiones, entre estas la odontología, desafortunadamente se encontró que de las 29 universidades de odontología, 4 públicas y 3 privadas no imparten este tipo de cursos, y en 10 universidades privadas y 6 públicas de postgrado tampoco.(Rodis et al., 2014)

El inglés con propósitos específicos ha sido tomado en cuenta para su implementación en el área médica dentro en la malla curricular de la carrera de Medicina en varias Universidades.(Isani, 2013; Kayaoğlu \& Dağ Akbaş, 2016; Pavel, 2014; Salager-Meyer, 2014; Valdés, González, Diaz, Verdayes, \& Diaz, 2010) El inglés es una herramienta indispensable para la actualización de conocimientos en el campo de la salud, pues permite entender la información de artículos científicos de cuartil 1 y 2 que únicamente se encuentran en inglés(Beltrán, 2006), la implementación del inglés con propósitos específicos dentro de la academia puede ser de gran contribución para el desarrollo de los futuros profesionales y mucho más en áreas de la salud, permitiendo el acceso a cientos de bases de información en el que el denominador común de la publicación científica es el idioma inglés.

\section{Metodología}

El proceso investigativo resultó del tipo exploratorio, de campo con un enfoque mixto. La población de estudio estuvo constituida por 26 docentes especialistas que imparten sus conocimientos a los estudiantes de la Carrera de Odontología de la Universidad Nacional de Chimborazo, a quienes se les entregó un cuestionario de 15 preguntas validado por el Alfa de Cronbach $(\alpha=0,872)$, cuyo valor fue mayor a 0,8 por lo que se puede indicar que el instrumento tuvo una buena consistencia interna y por lo tanto una fiabilidad aceptable.

Para ello el instrumento que valoró la percepción de los profesionales de esta rama, fue adaptado de fuentes bibliográficas relacionadas al inglés con propósitos médicos (Herrera \& Vallejo, 2013; Sierra-Galan, 2016; Weston, 2015); para los ítems que evaluaron la importancia del desarrollo de destrezas y dominios del idioma fueron valorados por una escala Likert con la siguiente escala de importancia: muy importante, importante, moderadamente importante, de poca importancia, sin importancia. Respecto al nivel de dominio la escala se determinó con las siguientes opciones: malo, regular, bueno, muy bueno, y excelente.

Para determinar las destrezas que bajo la percepción de los profesionales serían las adecuadas para el ámbito de la profesión se cuestionaron sobre las destrezas del idioma inglés como el escuchar, leer, 
hablar y escribir, considerando a los mismos en las diferentes aplicaciones en el ámbito de la profesión y formación.

Con el objetivo de caracterizar la percepción de la importancia que le conceden los profesionales del idioma inglés dentro de su profesión, y gracias a su experiencia se determinaron los temas apropiados para el desarrollo de la temática de inglés odontológico destinado al proceso de enseñanza de esta temática.

\section{Resultados y discusión}

Se evidenció que el $61 \%$ de docentes especialistas encuestados indicó que es muy importante la realización de preguntas y la participación en discusiones científicas en inglés dentro de su profesión. Se observó que los docentes odontólogos poseen falencias sobre las cuatro destrezas en el idioma inglés, pues solamente el 7,7\% manifestó tener un excelente nivel al escuchar y hablar, mientras que el 15, 4\% sobre el escribir y leer; por otro lado, el 61, $5 \%$ indicó que la lectura es la más necesaria para reforzar y actualizar sus conocimientos; más del 50\% consideró muy importante el escuchar, leer y hablar, en relación a los dominios y la importancia de las destrezas básicas del idioma inglés se puede notar que ninguno de los docentes considera a la escritura como una destreza de importancia; así mismo el escuchar y leer son los valores típicos de más frecuencia (Tabla 1). Finalmente, el número de páginas de lectura que realizan en una semana se determinó un rango entre 0 a 25 páginas al mes por parte de la mayoría de los docentes.

Tabla 1. Distribución de la población de estudio según destreza que considera más importante

\begin{tabular}{lcc}
\multicolumn{1}{c}{ Destreza } & Frecuencia absoluta & Porcentaje \\
\hline \hline Escuchar & 3 & 11,53 \\
Leer & 18 & 69,24 \\
Hablar & 5 & 19,23 \\
\hline Total & 26 & 100,00 \\
\hline \hline
\end{tabular}

Fuente: Elaboración propia

El nivel de dominio sobre la habilidad de leer considerada como la de mas alta importancia sobre el nivel de dominio mostró que los profesionales en un $38,46 \%$ consideran tener un dominio de lectura bueno y perciben que esta habilidad conlleva un nivel de muy importante en el desarrollo de la formación y profesión (Tabla 2).

Los temas más relevantes del área odontológica de acuerdo a los conocimientos, experiencia y necesidades de los docentes especialistas de la carrera de odontología son: educación y prevención en salud oral, patologías bucodentales más comunes, anatomía dental interna, diagnóstico pulpar y 
periapical, anatomía del periodonto, efectos del tabaquismo en la periodontitis crónica, técnicas de radiografía oral y puntos de referencia anatómicos, diagnóstico radiográfico de patologías dentales y maxilofaciales, pulpectomía, pulpotomía, cáncer oral, labio leporino y paladar hendido, exodoncia, complicaciones en cirugía oral, prótesis dental, diseño de prótesis parcial removible: conectores mayores y menores, maloclusión.

Tabla 2. Nivel de dominio de la destreza leer según la importancia que le concede a la población de estudio

Nivel de Nivel de importancia que se le concede a la habilidad de leer dominio de la destreza leer Poco importante Importante Muy importante

\begin{tabular}{lcccc}
\hline \hline Regular & - & 1 & 3 & 4 \\
Bueno & 1 & 1 & 10 & 12 \\
Muy bueno & - & 4 & 2 & 6 \\
Excelente & - & & 4 & 4 \\
\hline \multicolumn{1}{c}{ Total } & 1 & 6 & 19 & 26 \\
\hline \hline
\end{tabular}

Fuente: Elaboración propia

Para determinar el valor de significancia que demuestre que el domino promedio poblacional es superior al $70 \%$ como aceptable, se realizó la prueba T para una muestra mediante la significancia unilateral hacia la derecha, en la que se comparó si los dominios de los docentes superaron el 0,70 (p=0,00; 1 $\mathrm{p} / 2=1 ; \mathrm{t}=4,34(25))$ en cuyo caso se mostró un manejo de dominio menor al $70 \%$ destacando la necesidad de un texto para mejorar estas debilidades, la importancia de este aporte se mostró aceptable por parte de los docentes (Tabla 3). El uso del idioma inglés en el mundo científico se ha incrementado constantemente, la mayor parte de literatura especializada en las distintas áreas de la medicina es publicada en este idioma, es importante destacar que se ha convertido en el principal idioma de reuniones internacionales entre especialistas y conferencias de intercambio de conocimientos científicos, ya que las revistas más nombradas y conferencias más destacadas alrededor del mundo son dirigidas en inglés.(Allouche \& Benmoussat, 2012; Niazi, 2014) Lo que coincide con lo manifestado por la mayoría de docentes especialistas en este estudio, ya que ellos consideran muy importante compartir su conocimiento y experiencias en eventos académicos científicos internacionales sin tener como limitante el uso del idioma inglés.

Tabla 3. Significancia estadística prueba T de una muestra.

Valor de prueba $\mathbf{0} \mathbf{0 . 7 0}$

\begin{tabular}{lcccccc} 
& T & gl & $\begin{array}{c}\text { Sig. } \\
\text { (bilateral) }\end{array}$ & $\begin{array}{c}\text { Diferencia de } \\
\text { medias }\end{array}$ & $\begin{array}{c}\text { 95\% de intervalo de } \\
\text { confianza de la diferencia }\end{array}$ \\
\hline $\begin{array}{l}\text { Porcentaje } \\
\text { de Dominios }\end{array}$ & 4,346 & 25 & 0,00 & 0,12692 & 0,0668 & 0,1871 \\
\hline
\end{tabular}

Fuente: Elaboración propia 
Allouche propone que los estudiantes de medicina necesitan obtener conocimientos relacionados a su área por medio de la información disponible en conferencias, recursos multimedia, lectura de una variedad de material sobre ciencia y tecnología, además menciona la importancia de escribir y presentar oralmente reportes científicos, por esta razón propone la creación de un sílabo que atienda estas necesidades dentro de la enseñanza de inglés médico.(Allouche \& Benmoussat, 2012) El estudio mencionado anteriormente respalda la presente investigación, pues es de trascendental importancia determinar las necesidades reales que viven los docentes especialistas en interactuar y actualizar sus conocimientos odontológicos en el idiomas inglés, de ahí el requerimiento de construir un sílabo y desarrollar un libro de inglés con propósitos odontológicos que se adapte a las necesidades tanto de estudiantes como profesionales del área.

En el presente estudio el análisis de la importancia de se vio determinado a partir de la estimación de ésta en función de las destrezas del idioma como se muestra en la Tabla 1, de la misma forma se comprobó el valor significancia que demuestre que el valor de la medias de la importancia es mayor al valor aceptable de 0,70 considerando la prueba $T$ una vez determinado la distribución de datos normal $(\mathrm{p}=0,016)$, donde en valores estadísticos del porcentaje de importancia del desarrollo de destrezas supero el $82,69 \%(\mathrm{p}=0,045 ; \mathrm{p} / 2=0,0225 ; \mathrm{t}=4,1 ; \mathrm{gl}=25)$ y en los valores de significancia demostraron que se consideró importante el desarrollo de destrezas.

Javid en su estudio dirigido hacia las necesidades lingüísticas de los estudiantes determina que de acuerdo al nivel de suficiencia que los estudiantes consideran tener sobre las cuatro competencias básicas, la lectura es la que más dominan con una media de 2.26/5, seguida por el dominio sobre la escritura, el escuchar en tercer lugar y por último la destreza al hablar, lo que coincide con el presente estudio en donde se evidencia que la lectura es la destreza con mayor dominio por parte de los docentes de la carrera de odontología, con una media de 2,38/4, seguida por la escritura, en tercer lugar el escuchar y por último el hablar; al analizar la importancia que poseen las 4 competencias básicas. El estudio Javid (2011) indica que el escuchar es reportado como más importante con un media de 1,76/4, a diferencia del presente estudio donde se determina que la destreza considerada más importante es la lectura con una media de 2,69/3; por otro lado al analizar la importancia que tienen las diferentes actividades relacionadas a las cuatro competencias básicas en el estudio de Javid se determina que escuchar conferencias, realizar preguntas en una conferencia, leer libros y tomar apuntes de clases son las más importantes (Javid, 2011); mostrando una discrepancia entre los resultados del presente estudio que reporta la lectura de artículos de revistas, la comprensión de conferencias, la escritura de resúmenes de una investigación y la participación en discusiones científicas 
como las más importantes. En el estudio de Javid (2011) el número de páginas de lectura que realizan en una semana se determinó en un rango de 15 a 200 páginas indicando una diferencia considerable con la lectura de este estudio con rangos entre 0 a 25 páginas al mes por parte de los docentes.

Niazi reportó que se puede identificar la necesidad y la importancia de las destrezas del idioma, cuyos resultados indicaron que el $43 \%$ de profesores y estudiantes consideraron poseer los mismos, se destaca que el inglés es usado en un 91 al $100 \%$ en sus estudios en medicina y al trabajo dentro de la profesión, por otro lado los profesores de medicina mencionan que frecuentemente necesitan el inglés para varios propósitos durante su etapa académica y profesional, como: participación en seminarios, lectura de literatura en su especialidad, conversaciones, actualización de conocimientos sobre nuevas técnicas y tratamientos, para entender manuales para el manejo de equipos, realizar conferencias, escribir reportes de laboratorio, recetas médicas, entre otras (Niazi, 2014), estos resultados coinciden con la importancia que los docentes muestran ante estas actividades en el presente estudio debido a que el $50 \%$ de especialistas cataloga entre importante y muy importante a cada una de estas actividades.

Gerivani en su estudio revela que para los estudiantes la destreza de la lectura es de vital importancia(Gerivani, 2016), que en relación con el presente estudio coincide en este criterio donde la población constituida por docentes especialistas indicó en un 73,1\% como muy importante la lectura en el ámbito de su profesión.

Como lo indica Herrera en su estudio de evaluación de aprendizajes de inglés y su transversalidad en el programa de psicología el dominio de habilidades de escritura, lectura, escuchar y hablar en el idioma inglés fue del $25 \%$ o menos demostrando su poca habilidad del manejo del idioma inclusive comparados con una base de datos reportados de estudiantes de ingreso a la universidad, en cuyo caso destacan la importancia de aprender el mismo como parte fundamental de su formación.(Herrera \& Vallejo, 2013) En el caso de la presente investigación orientada a docentes el $50 \%$ o menos de la población de estudio indicó que no manejar la destreza de escuchar en su práctica profesional considerando el valor de 0,70 como aceptable, en el caso del dominio de lectura el $100 \%$ indicó tener un porcentaje de dominio general por debajo de lo aceptable y en el caso de dominio del habla el 75\% de ellos están por debajo del valor aceptable, el dominio de la escritura no es considerado de importancia.

\section{Conclusiones}

Se evidencia que los docentes especialistas poseen falencias sobre las cuatro destrezas del idioma inglés, determinado que tan solo el $25 \%$ presentó un porcentaje aceptable de manejo de éstas, por lo que se convierte en una 
limitante al momento de asistir o participar en eventos académicos científicos desarrollados en este idioma anglosajón.

El idioma inglés fue reportado como muy importante por más de la mitad de los especialistas dentro de todas las actividades que involucran las cuatro destrezas básicas del este idioma, como un medio que permite la actualización y refuerzo de conocimientos sobre el área de especialización odontológica de interés.

Se concluye que la necesidad prioritaria de construir un libro de Inglés con Propósitos Odontológicos, que sirva como una herramienta de trabajo, el cual debería constar de al menos 17 lecciones con los temas determinados a través de este estudio incluyendo las cuatro destrezas básicas del idioma inglés.

\section{References:}

1. Allouche, W.-S., \& Benmoussat, S. (2012). Designing and Implementing an ESP Course for Medical Students: A Step Towards Building up a Knowledge Society. Abou-Berk Belkaid University.

2. Beltrán, Ó. (2006). Factor de impacto. Revista Colombiana de Gastroenetrología, 21(1), 57-61.

3. EF Education First. (2018). EF EPI Índice del Dominio del Inglés de EF 2018. Retrieved from https://www.ef.com/_/ /media/centralefcom/epi/downloads/fullreports/v8/ef-epi-2018-spanish-latam.pdf

4. Gerivani, L. (2016). An Analysis of the English Language Needs of Medical Students and General Practitioners : A Case Study of Guilan University of Medical Sciences. International Journal of English Language and Literature Studies, 5(2), 104-110. https://doi.org/10.18488/journal.23/2016.5.2/23.2.104.110

5. Herrera, J., \& Vallejo, A. (2013). Evaluación del aprendizaje de Inglés y su transversalidad en el programa de Psicología. Universidad Veracruzana.

6. Isani, S. (2013). Brian Paltridge, Sue Starfield (eds.), The Handbook of English for Specific Purposes. ASp [Online].

7. Javid, C. Z. (2011). EMP Needs of Medical Undergraduates in a Saudi Context. Kashmir Journal of Language Research, 14(1), 89-110.

8. Kayaoğlu, M. N., \& Dağ Akbaş, R. (2016). An Investigation into Medical Students' English Language Needs. Participatory Educational Research, spi16(1), 63-71. https://doi.org/10.17275/per.16.spi.1.8

9. Montes De Oca, I., \& Morete, C. (2017). Diseño de la estrategia de Inglés con propósitos específicos en un contexto extracurricular para el aprendizaje del Inglés técnico médico en estudiantes de medicina de la Universidad Regional Autónoma de los Andes. Pontificia 
Universidad Católica del Ecuador Sede Ambato. Retrieved from http://repositorio.pucesa.edu.ec/handle/123456789/2138

10. Niazi, M. M. (2014). The Need for English Language Courses in Pakistani Medical Colleges. New Horizons, 8(1), 39-52.

11. Pavel, E. (2014). Teaching English for medical purposes. Bulettin of the Transilvania University of Brasov, Series VII: Social Sciences and Law, (2), 39-46.

12. Rodis, O. M. M., Barroga, E., Barron, J. P., Hobbs, J., Jayawardena, J. A., Kageyama, I.,Yoshimoto, K. (2014). A proposed core curriculum for dental English education in Japan. BMC Medical Education, 14(1), 1-9. https://doi.org/10.1186/s12909-014-0239-4

13. Rozgienè, I., \& Trečiokaite, A. (2019). Survey of the English Language Needs of the Senior Students of Physical and Biomedical Sciences.Verbum,2,122-130. https://doi.org/10.15388/verb.2011.2.4962

14. Salager-Meyer, F. (2014). Origin and development of English for Medical Purposes. Part II: Research on spoken medical English. MedicalWriting,23(2),129-131. https://doi.org/10.1179/2047480614z.000000000204

15. Sequeira, A. H. (2016). Introduction to concepts of teaching and learning. Applied and Social Sciences Magazine, 6(1), 1-6. Recuperadodehttps://papers.ssrn.com/sol3/papers.cfm?abstract_id=21 50166

16. Sierra-Galan, L. (2016). El idioma « inglés » en la medicina. Archivos de Cardiología de México,86(1),97-98. https://doi.org/http://dx.doi.org/10.1016/j.acmx.2015.09.001

17. Valdés, M., González, S., Diaz, I., Verdayes, A., \& Diaz, L. (2010). La enseñanza del inglés en las ciencias médicas : su repercusión social The teaching of English Language in Medical Studies : its social. Rev. Ciencias Médicas. Julio, 14(3). Retrieved from http://scielo.sld.cu/pdf/rpr/v14n3/rpr06310.pdf

18. Weston, J. (2015). How Much English Does a Dentist Need ? National Council of Teachers of English, 11(2), 71-74. Retrieved from http://www.jstor.org/stable/355966 